\title{
HYPOCOERCIVITY FOR LINEAR KINETIC EQUATIONS CONSERVING MASS
}

\author{
JEAN DOLBEAULT, CLÉMENT MOUHOT, AND CHRISTIAN SCHMEISER
}

\begin{abstract}
We develop a new method for proving hypocoercivity for a large class of linear kinetic equations with only one conservation law. Local mass conservation is assumed at the level of the collision kernel, while transport involves a confining potential, so that the solution relaxes towards a unique equilibrium state. Our goal is to evaluate in an appropriately weighted $L^{2}$ norm the exponential rate of convergence to the equilibrium. The method covers various models, ranging from diffusive kinetic equations like VlasovFokker-Planck equations, to scattering models or models with time relaxation collision kernels corresponding to polytropic Gibbs equilibria, including the case of the linear Boltzmann model. In this last case and in the case of VlasovFokker-Planck equations, any linear or superlinear growth of the potential is allowed.
\end{abstract}

\section{Method, Result And COnsequences}

1.1. Linear kinetic equations and hypocoercivity. We consider linear kinetic equations which can be written as

$$
\partial_{t} f+\mathrm{T} f=\mathrm{L} f, \quad f=f(t, x, v), \quad(t, x, v) \in \mathbb{R}^{+} \times \mathbb{R}^{d} \times \mathbb{R}^{d}, \quad d \in \mathbb{N},
$$

and describe the evolution of a distribution function $f$. The transport operator

$$
\mathrm{T}:=v \cdot \nabla_{x}-\nabla_{x} V \cdot \nabla_{v}
$$

has characteristics given on the phase space $\mathbb{R}^{d} \times \mathbb{R}^{d}$ by the flow of the Hamiltonian

$$
(x, v) \mapsto E(x, v):=\frac{1}{2}|v|^{2}+V(x) .
$$

The external potential $V=V(x)$ is a measurable function on $\mathbb{R}^{d}$. The collision operator $\mathrm{L}$ is independent of time $t$ and acts as a multiplicator in the position variable $x$. The variable $v$ is the velocity.

We shall consider steady states which are in the intersection of the null spaces of $T$ and $L$ simultaneously. We shall assume that there exists a nonnegative energy profile function $\Gamma$ such that, for each fixed value of $x$, the nullspace $\mathcal{N}(\mathrm{L})$ of $\mathrm{L}$ is spanned by $F(x, v):=\Gamma(E(x, v))$, so that

$$
\mathcal{N}(\mathrm{L})=\{f(x, v): \exists \phi(x) \text { such that } f(x, v)=\phi(x) F(x, v)\} .
$$

Received by the editors May 10, 2010 and, in revised form, November 11, 2012.

2010 Mathematics Subject Classification. Primary 82C40; Secondary 35B40, 35F10, 35H10, 35H99, 76P05.

Key words and phrases. Kinetic equations, hypocoercivity, Boltzmann, BGK, relaxation, diffusion limit, nonlinear diffusion, Fokker-Planck, confinement, spectral gap, Poincaré inequality, Hardy-Poincaré inequality. 
Functions in $\mathcal{N}(\mathrm{L})$ are local equilibria; they depend on $x$ and $t$. The function $F$ is a global equilibrium or global Gibbs state. It is independent of $t$ (stationary) and isotropic with respect to $v$. Consistently, we shall further assume that $L$ has rotational symmetry in $v$, i.e. $\mathrm{R}_{v} \mathrm{~L}=\mathrm{L} \mathrm{R}_{v}$ for any rotation operator $\mathrm{R}_{v}$ acting on the velocity space. Under the assumption that the support of $F$ is connected, the intersection of the null spaces of $\mathrm{L}$ and $\mathrm{T}$ is generated by $F$. We assume from now on that $F$ is strictly positive, integrable, and normalized by

$$
\iint_{\mathbb{R}^{d} \times \mathbb{R}^{d}} F \mathrm{~d} v \mathrm{~d} x=1
$$

We shall refer to this assumption as Assumption (H0) and assume that it holds throughout the paper, although we shall not specify it explicitly when it is not useful for the understanding of our arguments. Under such a normalization condition, we shall prove that $F$ is the unique stationary distribution function. Integrability with respect to $v$ is an assumption on $\Gamma$, whereas integrability with respect to $x$ requires a $\Gamma$-dependent growth of the external potential $V$. Such a property is a confinement condition.

The one-dimensionality of $\mathcal{N}(\mathrm{L})$ for fixed $x$ suggests the existence of one local (in $x$ ) conservation law. We shall therefore assume the local conservation of mass, that is

$$
\int_{\mathbb{R}^{d}} \mathrm{~L} f \mathrm{~d} v=0
$$

for any sufficiently smooth and sufficiently fast decaying distribution $f$. Global mass conservation for solutions of (1.1) follows:

$$
\frac{\mathrm{d}}{\mathrm{d} t} \iint_{\mathbb{R}^{d} \times \mathbb{R}^{d}} f \mathrm{~d} v \mathrm{~d} x=\iint_{\mathbb{R}^{d} \times \mathbb{R}^{d}}(\mathrm{~L}-\mathrm{T}) f \mathrm{~d} v \mathrm{~d} x=0 .
$$

For an integrable initial datum

$$
f(t=0, \cdot, \cdot)=f_{I},
$$

let $M:=\iint_{\mathbb{R}^{d} \times \mathbb{R}^{d}} f_{I} \mathrm{~d} v \mathrm{~d} x$, so that $M F$ is the unique global Gibbs state with mass $M$. In this paper we investigate the asymptotic behavior of the semigroup generated by $\mathrm{L}-\mathrm{T}$. Our goal is to quantify its stability or, to be precise, to determine the rate of convergence of $f$ towards $M F$ as $t \rightarrow \infty$. Since the equation is linear, there is no restriction to study fluctuations around a global equilibrium, that is, solutions $f$ of (1.1) which satisfy

$$
M=\iint_{\mathbb{R}^{d} \times \mathbb{R}^{d}} f(t, x, v) \mathrm{d} v \mathrm{~d} x=\iint_{\mathbb{R}^{d} \times \mathbb{R}^{d}} f_{I}(x, v) \mathrm{d} v \mathrm{~d} x=0 .
$$

Notice that distribution functions are usually nonnegative, but fluctuations around an equilibrium have to change sign.

Local mass conservation for $f$ and $F$ imply the identity

$$
\int_{\mathbb{R}^{d}} \mathrm{~L} f\left(\frac{f}{F}\right) \mathrm{d} v=\int_{\mathbb{R}^{d}} \mathrm{~L}(f-\phi F)\left(\frac{f-\phi F}{F}\right) \mathrm{d} v
$$

for any function $\phi=\phi(x)$, thus showing that the left hand side is, at least formally, quadratic in the distance between $f$ and the kernel of $\mathrm{L}$. This suggests we introduce the space $L^{2}(\mathrm{~d} \mu)$, where the measure $\mathrm{d} \mu$ is defined on the phase space by

$$
\mathrm{d} \mu=\mathrm{d} \mu(x, v):=\frac{\mathrm{d} v \mathrm{~d} x}{F(x, v)}, \quad(x, v) \in \mathbb{R}^{d} \times \mathbb{R}^{d} .
$$


We shall denote by $\langle\cdot, \cdot\rangle$ the corresponding scalar product and by $\|\cdot\|$ the associated norm, so that $\langle f, g\rangle=\iint_{\mathbb{R}^{d} \times \mathbb{R}^{d}} f g \mathrm{~d} \mu$ and $\|f\|^{2}=\langle f, f\rangle$. The orthogonal projection $\Pi$ on the set of local equilibria is denoted by

$$
\Pi f:=\frac{\rho_{f}}{\rho_{F}} F, \quad \text { with } \rho_{f}:=\int_{\mathbb{R}^{d}} f \mathrm{~d} v .
$$

We also assume that the collision operator is dissipative in the sense that an ' $H$ theorem' holds, i.e. $\langle\mathrm{L} f, f\rangle \leq 0$. Since the transport operator $\mathrm{T}$ is skew symmetric with respect to $\langle\cdot, \cdot\rangle$, this implies the entropy inequality

$$
\frac{1}{2} \frac{\mathrm{d}}{\mathrm{d} t}\|f\|^{2}=\langle\mathrm{L} f, f\rangle \leq 0 .
$$

Well posedness of the initial value problem for (1.1) with initial data $f_{I} \in L^{2}(\mu)$ is a consequence of maximal dissipativity of $L-T$, which will be assumed in the following. For the examples of $L$ considered in Sections 3 and 4 below, this result has been proven (see [22, 35] for Fokker-Planck operators) or it is immediate (for BGK operators being bounded perturbations of the transport operator $\mathrm{T}$ ).

Under the normalization condition (1.2), if the entropy dissipation $-\langle\mathrm{L} f, f\rangle$ was coercive with respect to the norm $\|\cdot\|$, exponential decay to zero as $t \rightarrow \infty$ would follow. However such a coercivity property cannot hold since $L$ vanishes on the set of local equilibria. Instead we shall assume that microscopic coercivity holds, i.e. there exists a positive constant $\lambda_{m}$, such that

$$
-\langle\mathrm{L} f, f\rangle \geq \lambda_{m}\|(1-\Pi) f\|^{2} \quad \text { for all } f \in D(\mathrm{~L}) .
$$

The key tool of our method is a modified entropy functional $\mathrm{H}[f]$, whose square root is a norm equivalent to $\|\cdot\|$, such that

$$
\frac{\mathrm{d}}{\mathrm{d} t} \mathrm{H}[f] \leq-\lambda \mathrm{H}[f],
$$

for an explicitly computable positive constant $\lambda$. As a consequence, we find an estimate of the exponential decay rate of the semigroup. Following the vocabulary used in 21, 28, 35, such a strategy will be called hypocoercivity.

In some cases, the existence of a spectral gap can be obtained by nonconstructive compactness methods; see for instance 34 in the case of the linearized Boltzmann equation on the torus. For a maximal dissipative operator $U$ with a spectral gap $\lambda>0$, it is well known (see [29]) that there exists a norm equivalent to the ambient norm, for which the semigroup of $U+\lambda$ is contractive. However this method is not constructive regarding the norm of contractivity and gives no estimate on $\lambda$. In our approach, under assumptions specifically adapted to kinetic theory, we are able to construct an explicit Hilbert norm which is equivalent to the standard norm of $L^{2}(\mathrm{~d} \mu)$ and to estimate $\lambda$.

Various results related to hypocoercivity have recently appeared, on large time estimates [5,8,9,13]; based on hypoellipticity [20,22,23]; on hypocoercivity itself [21, 28, 35]; on applications of the so-called kinetic-fluid decomposition [15, 19, 31, 33; on hyperbolic estimates based on micro-macro decompositions [25, 27, 36]. Some of the results of this paper, namely Theorems 9 and 14, have been announced in 12 . without complete proofs.

Our purpose is to establish, in a simplified framework, sufficient conditions for proving hypocoercivity for a large class of linear kinetic models confined by an external potential, without assuming regularity on the initial datum and valid for 
hypoelliptic kinetic Fokker-Planck equations as well as singularity preserving collisional kinetic equations. This is the main difference with hypoelliptic methods. The method also makes use of a micro-macro decomposition. Accordingly we shall split our assumptions into two main requirements: microscopic coercivity as introduced above, and a macroscopic coercivity assumption, which is a spectral gap-like inequality for the operator obtained when taking an appropriate macroscopic diffusion limit that we shall now describe.

1.2. Formal macroscopic limit. As a motivation for the macroscopic coercivity assumption, we recall, at a formal level, the macroscopic diffusion limit procedure, which can be seen as intermediate asymptotics governing the long time behaviour of solutions. On a large time scale, it heuristically models how local equilibria relax towards the global Gibbs state. Since the macroscopic flux of the equilibrium distribution vanishes, i.e. $\int_{\mathbb{R}^{d}} v F \mathrm{~d} v=0$, the appropriate macroscopic rescaling of the solution of (1.1) is given by

$$
f^{\varepsilon}(t, x, v)=f\left(\varepsilon^{2} t, \varepsilon x, v\right), \quad 0<\varepsilon \ll 1,
$$

which is known as the parabolic rescaling. In terms of the operators introduced above, the vanishing flux condition can be written as $\Pi$ Т $\Pi=0$, which will have to be assumed in the abstract framework of Section 1.4 below (see Assumption (H3)).

Assuming that the potential $V$ is rescaled accordingly, we obtain the singular limit problem

$$
\varepsilon^{2} \partial_{t} f^{\varepsilon}+\varepsilon \mathrm{T} f^{\varepsilon}=\mathrm{L} f^{\varepsilon}
$$

as $\varepsilon \rightarrow 0$. The assumption $\lim _{\varepsilon \rightarrow 0} f^{\varepsilon}=f^{0}$ leads to $\mathrm{L} f^{0}=0$ and, thus, $f^{0}=\Pi f^{0}$. The identities $\Pi \mathrm{L}=\mathrm{L} \Pi=\Pi \mathrm{T} \Pi=0$ imply the relations

$$
\varepsilon \partial_{t} f^{\varepsilon}+\mathrm{T} f^{\varepsilon}=\mathrm{L} R^{\varepsilon}, \quad \partial_{t} \Pi f^{\varepsilon}+\Pi \mathrm{T} R^{\varepsilon}=0,
$$

with $R^{\varepsilon}:=\frac{1}{\varepsilon}(1-\Pi) f^{\varepsilon}$. Assuming formally that $\lim _{\varepsilon \rightarrow 0} R^{\varepsilon}=R^{0}$, the first equation can be solved for $\varepsilon=0$ with respect to $R^{0}$, giving

$$
R^{0}=\mathrm{J \top} f^{0}=\mathrm{J} \mathrm{T} \Pi f^{0},
$$

where $J$ denotes the inverse of the restriction of $L$ to the orthogonal complement of its null space. Note that the inhomogeneity $\mathrm{\top} \Pi f^{0}$ satisfies the solvability condition $\Pi \mathrm{\top} \Pi f^{0}=0$. The second equation becomes

$$
\partial_{t} \Pi f^{0}=(\mathrm{T} \Pi)^{*} \mathrm{~J}(\mathrm{~T} \Pi) f^{0},
$$

where the superscript $*$ denotes the adjoint operator with respect to $\langle\cdot, \cdot\rangle$, and the skew symmetry of $T$ has been used. A straightforward computation shows that this is equivalent to a drift-diffusion equation for the macroscopic density $\rho^{0}=\rho_{f^{0}}$ :

$$
\partial_{t} \rho^{0}=\nabla_{x} \cdot\left[\rho_{F} \sigma \nabla_{x}\left(\frac{\rho^{0}}{\rho_{F}}\right)\right]=\nabla_{x} \cdot\left[\nabla_{x}\left(\sigma \rho^{0}\right)+\gamma \rho^{0} \nabla_{x} V\right] .
$$

Here $\sigma$ is scalar due to the rotational symmetry of $\mathrm{L}$,

$$
\rho_{F} \sigma=-\frac{1}{d} \int_{\mathbb{R}^{d}} v \cdot \mathrm{J}(v F) \mathrm{d} v \quad \text { and } \quad \gamma \nabla_{x} V=-\frac{1}{\rho_{F}} \nabla_{x}\left(\rho_{F} \sigma\right),
$$

with the operator $\mathrm{J}$ being negative definite on $(1-\Pi) L^{2}(\mathrm{~d} \mu), \sigma(x)>0$ for all $x \in \mathbb{R}^{d}$. In the two following important cases, the macroscopic transport coefficients $\gamma$ and $\sigma$ have particularly simple expressions. 
Case (C1). When $\Gamma(s)=e^{-s}$, the global Gibbs state is a Maxwellian or Gaussian function, which factorizes as

$$
F(x, v)=\rho_{F}(x) \mathfrak{M}(v), \quad \text { with } \quad \rho_{F}=\frac{e^{-V}}{\int_{\mathbb{R}^{d}} e^{-V} \mathrm{~d} x} \quad \text { and } \quad \mathfrak{M}(v)=\frac{e^{-|v|^{2} / 2}}{(2 \pi)^{d / 2}} .
$$

Notice that the separation of position and velocity variables is a characteristic property of Maxwellian functions. Both coefficients $\gamma$ and $\sigma$ are constant, equal to $\frac{1}{d} \int_{\mathbb{R}^{d}} v \cdot \mathrm{J}(v \mathfrak{M}) \mathrm{d} v$ and $\rho^{0}$ solves the Fokker-Planck equation

$$
\partial_{t} \rho^{0}=\sigma \nabla_{x} \cdot\left(\nabla_{x} \rho^{0}+\rho^{0} \nabla_{x} V\right) .
$$

Case (C2). The collision operator $\mathrm{L}$ is, for fixed $x$, a time-relaxation operator onto $\operatorname{span}\{F\}$, i.e.,

$$
\mathrm{L}=\Pi-1 .
$$

In this case $\mathrm{J}=-\mathrm{Id}$ holds, so that

$$
\rho_{F} \sigma=m_{F}:=\frac{1}{d} \int_{\mathbb{R}^{d}}|v|^{2} F \mathrm{~d} v
$$

and, since $\nabla_{x}\left(\rho_{F} \sigma\right)=-\rho_{F} \nabla_{x} V$ :

$$
\begin{aligned}
& \nabla_{x}\left(\frac{1}{d} \int_{\mathbb{R}^{d}}|v|^{2} F \mathrm{~d} v\right)=\frac{\nabla_{x} V}{d} \int_{\mathbb{R}^{d}}|v|^{2} \Gamma^{\prime}(E(x, v)) \mathrm{d} v \\
& \quad=\frac{\nabla_{x} V}{d} \int_{\mathbb{R}^{d}} v \cdot \nabla_{v} \Gamma(E(x, v)) \mathrm{d} v=\frac{\nabla_{x} V}{d} \int_{\mathbb{R}^{d}} v \cdot \nabla_{v} F \mathrm{~d} v=-\rho_{F} \nabla_{x} V
\end{aligned}
$$

(recall in this calculation that $E(x, v)=|v|^{2} / 2+V(x)$ ), the macroscopic limit equation reads

$$
\partial_{t} \rho^{0}=\nabla_{x} \cdot\left(\nabla_{x}\left(\sigma \rho^{0}\right)+\rho^{0} \nabla_{x} V\right) .
$$

The intersection of both cases, (C1) and (C2), i.e. $\mathrm{L}=\Pi-1$ with $\Pi f=\rho_{f} \mathfrak{M}$, gives $\sigma=\gamma=1$. This is the linear BGK case, which has been considered in [12]. In both cases, (1.3) can be rewritten as

$$
\partial_{t} \Pi f^{0}=-\sigma_{0}(\mathrm{~T} \Pi)^{*}(\mathrm{~T} \Pi) f^{0}
$$

for some positive constant $\sigma_{0}$, with $\sigma_{0} \equiv \sigma$ in Case (C1) and $\sigma_{0}=1$ in Case (C2). With assumption (1.2) on the initial data, we expect decay to zero of the solution. Under a macroscopic coercivity assumption, namely (H2) (see below), which is equivalent to a Poincaré inequality (see Lemma 3), the decay of $\Pi f^{0}$ is exponential.

1.3. Method and main result in an abstract setting. We start with the basic assumption that $L$ and $T$ are closed linear operators on a Hilbert space $\mathcal{H}$, such that $\mathrm{L}-\mathrm{T}$ generates the strongly continuous semigroup $e^{(\mathrm{L}-\mathrm{T}) t}$ on $\mathcal{H}$. The orthogonal projection on the null space $\mathcal{N}(\mathrm{L})$ of $\mathrm{L}$ is denoted by $\Pi$ and $\mathcal{D}(\mathrm{L})$ is the domain of $L$. We assume that the restriction of $L$ to $\mathcal{N}(L)^{\perp}$ is coercive. More precisely, our first assumption is:

Assumption (H1) (microscopic coercivity): The operator $\mathrm{L}$ is symmetric and there exists $\lambda_{m}>0$ such that

$$
-\langle\mathrm{L} f, f\rangle \geq \lambda_{m}\|(I-\Pi) f\|^{2} \quad \text { for all } f \in \mathcal{D}(\mathrm{L}) .
$$


Motivated by the results of Section [1.2, coercivity of the transport operator is required, when acting on $\mathcal{N}(\mathrm{L})$ :

Assumption (H2) (macroscopic coercivity): The operator $\mathrm{T}$ is skew symmetric and there exists $\lambda_{M}>0$ such that

$$
\|\mathrm{T} \Pi f\|^{2} \geq \lambda_{M}\|\Pi f\|^{2} \quad \text { for all } f \in \mathcal{H} \text { such that } \Pi f \in \mathcal{D}(\mathrm{T}) .
$$

Inspired by [21, we introduce the modified entropy

$$
\mathrm{H}[f]:=\frac{1}{2}\|f\|^{2}+\varepsilon\langle\mathrm{A} f, f\rangle, \quad \text { with } \mathrm{A}:=\left(1+(\mathrm{T} \Pi)^{*}(\mathrm{~T} \Pi)\right)^{-1}(\mathrm{~T} \Pi)^{*} .
$$

The constant $\varepsilon>0$ will be chosen below. A straightforward computation for a solution $f$ of (1.1), now considered as an abstract ODE, gives

$$
\frac{\mathrm{d}}{\mathrm{d} t} \mathrm{H}[f]=-\mathrm{D}[f]
$$

where the dissipation of entropy functional is given by

$$
\mathrm{D}[f]:=-\langle\mathrm{L} f, f\rangle+\varepsilon\langle\mathrm{AT} \Pi f, f\rangle+\varepsilon\langle\mathrm{AT}(1-\Pi) f, f\rangle-\varepsilon\langle\mathrm{TA} f, f\rangle-\varepsilon\langle\mathrm{AL} f, f\rangle .
$$

We have used here that $L A=0$ which follows from $A=\Pi A$. The latter identity is obtained by writing $\mathrm{A} f=g$ as

$$
(\mathrm{T} \Pi)^{*} f=g+(\mathrm{T} \Pi)^{*}(\mathrm{~T} \Pi) g \quad \Longleftrightarrow \quad g=-\Pi \mathrm{\top} f+\Pi \mathrm{T}^{2} \Pi g \in \Pi \mathcal{H} .
$$

By (H1), (H2), and by A T $\Pi=\left(1+(\mathrm{T} \Pi)^{*}(\mathrm{~T} \Pi)\right)^{-1}(\mathrm{~T} \Pi)^{*}(\mathrm{~T} \Pi)$, the sum of the first two terms in $\mathrm{D}[f]$ is coercive:

$$
-\langle\mathrm{L} f, f\rangle+\varepsilon\langle\mathrm{AT} \Pi f, f\rangle \geq \min \left\{\lambda_{m}, \frac{\varepsilon \lambda_{M}}{1+\lambda_{M}}\right\}\|f\|^{2} .
$$

For the completion of our program, we need to show that $\mathrm{H}[f]$ is equivalent to $\|f\|^{2}$ and to control the last three terms of $\mathrm{D}[f]$. Part of this can be carried out at the abstract level under the following additional assumption:

\section{Assumption (H3):}

$$
\Pi \mathrm{\top} \Pi=0 .
$$

Lemma 1. Let assumption (H3) hold. Then the operators A and T A are bounded, and for all $f \in \mathcal{H}$,

$$
\|\mathrm{A} f\| \leq \frac{1}{2}\|(1-\Pi) f\| \quad \text { and } \quad\|\mathrm{T} \mathrm{A} f\| \leq\|(1-\Pi) f\| .
$$

Proof. The equation A $f=g$ is equivalent to

$$
(\mathrm{T} \Pi)^{*} f=g+(\mathrm{T} \Pi)^{*}(\mathrm{~T} \Pi) g .
$$

Writing this as $g=\Pi \mathrm{T}^{2} \Pi g-\Pi \mathrm{T} f$ proves $\mathrm{A}=\Pi \mathrm{A}$ and, thus, $\mathrm{TA} f=\mathrm{T} \Pi g$. Taking the scalar product of the above equality with $g$ and using (H3), we get

$$
\begin{aligned}
\|g\|^{2}+\|\mathrm{\top} \Pi g\|^{2} & =\langle f, \mathrm{\top} \Pi g\rangle=\langle(1-\Pi) f, \mathrm{\top} \Pi g\rangle \\
& \leq\|(1-\Pi) f\|\|\mathrm{T} \Pi g\| \leq \frac{1}{4}\|(1-\Pi) f\|^{2}+\|\mathrm{\top} \Pi g\|^{2},
\end{aligned}
$$

which completes the proof. 
The boundedness of the remaining terms in $\mathrm{D}[f]$ has to be proven case by case. We shall therefore assume it in the abstract setting.

Assumption (H4) (Boundedness of auxiliary operators): The operators A T $(1-\Pi)$ and $\mathrm{A} L$ are bounded, and there exists a constant $C_{M}>0$ such that, for all $f \in \mathcal{H}$,

$$
\|\mathrm{AT}(1-\Pi) f\|+\|\mathrm{AL} f\| \leq C_{M}\|(1-\Pi) f\| \text {. }
$$

Theorem 2. Let assumptions (H1)-(H2)-(H3)-(H4) hold. Then there exist positive constants $\lambda$ and $C$, which are explicitly computable in terms of $\lambda_{m}, \lambda_{M}$, and $C_{M}$, such that, for any initial datum $f_{I} \in \mathcal{H}$,

$$
\left\|e^{t(\mathrm{~L}-\mathrm{T})} f_{I}\right\| \leq C e^{-\lambda t}\left\|f_{I}\right\|, \quad \forall t \geq 0 .
$$

Proof. The first inequality in (1.5) implies

$$
\frac{1}{2}(1-\varepsilon)\|f\|^{2} \leq \mathrm{H}[f] \leq \frac{1}{2}(1+\varepsilon)\|f\|^{2} .
$$

For any $\varepsilon \in(0,1), \mathrm{H}[f]$ is equivalent to $\|f\|^{2}$. The second inequality in (1.5) and (H1)-(H2)-(H3)-(H4) imply

$$
\begin{aligned}
\mathrm{D}[f] & \geq \lambda_{m}\|(1-\Pi) f\|^{2}+\frac{\varepsilon \lambda_{M}}{1+\lambda_{M}}\|\Pi f\|^{2}-\varepsilon\left(1+C_{M}\right)\|(1-\Pi) f\|\|f\| \\
& \geq\left[\lambda_{m}-\varepsilon\left(1+C_{M}\right) \frac{1}{2 \delta}\right]\|(1-\Pi) f\|^{2}+\varepsilon\left[\frac{\lambda_{M}}{1+\lambda_{M}}-\left(1+C_{M}\right) \frac{\delta}{2}\right]\|\Pi f\|^{2}
\end{aligned}
$$

for an arbitrary positive $\delta$. By choosing first $\delta$ and then $\varepsilon$ small enough, a positive constant $\kappa$ can be found, such that $\mathrm{D}[f] \geq \kappa\|f\|^{2}$. Using (1.6), this implies

$$
\frac{\mathrm{d}}{\mathrm{d} t} \mathrm{H}[f] \leq-\frac{2 \kappa}{1+\varepsilon} \mathrm{H}[f],
$$

for $f=e^{t(\mathrm{~L}-\mathrm{T})} f_{I}$, completing the proof with $\lambda=\kappa /(1+\varepsilon)$ and $C=\sqrt{\frac{1+\varepsilon}{1-\varepsilon}}$.

Let us conclude this abstract approach by some comments. First of all, our proof is constructive: $\mathrm{H}$ is an explicit Lyapunov functional and $\lambda$ can be computed. The work of F. Hérau has been a crucial source of inspiration for our method. In [21, he deals with the linear time relaxation collision kernel corresponding to Maxwellian Gibbs states, in case of a confining potential $V$ growing at most quadratically at infinity and such that the associated Witten Laplacian satisfies a spectral gap inequality. In our approach, we are able to relax some of these assumptions; see Theorem 9 ,

Our results apply to various Fokker-Planck and Boltzmann models. We shall compare applications of Theorem 2 to previous results in Section 3. Only [28] and [35] deal with abstract results like the ones of Theorem 2. Ours are more general than the ones of [28] since we deal with a general confining potential. In [28], the problem is indeed set on a torus, a setting to which our method can be adapted without any difficulty. It is also more general than in [35] since we deal not only with Fokker-Planck type operators, or operators in Hörmander form in the words of [35], but also with nonlocal integral collision operators, like in [28. Last but not least, our results are also stronger than those in 28] and 35] in the sense that we construct a zeroth order norm of hypocoercivity, which is equivalent to $L^{2}$ and not $H^{k}$, for some $k \geq 1$. However, our results are weaker than those in [28] at least in one aspect: we only deal with models with 1-dimensional space of collision invariants, whereas, in [28, any finite dimension is allowed. It is an interesting question to 
extend our approach to such a situation, however for a general confinement there are serious new difficulties of a geometric nature; this is the purpose of a current research project [10].

1.4. Hypocoercivity for a toy problem. To illustrate the fact that our formal setting applies to other models than the kinetic equations of Section 1.1, we introduce the following toy model, which captures very well the essential features of our hypocoercive approach. We consider a one-dimensional Cattaneo model introduced in [6], which can be written as a kinetic model with only two velocities $v= \pm 1$, and where $L$ describes a switching process between the two velocities without preference for one of them. As a further simplification we replace the confining potential by a periodicity assumption, where $x$ varies in a one-dimensional torus. The model equations are

$$
\partial_{t} f^{ \pm} \pm \partial_{x} f^{ \pm}= \pm \frac{1}{2}\left(f^{-}-f^{+}\right)
$$

for the distributions $f^{ \pm}(t, x)$ of right- and left-moving particles, periodic in $x$ with period $2 \pi$.

The interest of such a model is that it gives an application of our hypocoercivity method in a discrete setting, or even for a finite dimensional ODE version of it, if we truncate the Fourier sum in the $x$ variable and keep only a finite number of terms.

Initial value problems can be solved explicitly by Fourier decomposition. Introducing the total density $\rho=f^{+}+f^{-}$, the total flux $j=f^{+}-f^{-}$, and their Fourier representations

$$
\rho(t, x)=\sum_{k \in \mathbb{Z}} \rho_{k}(t) e^{i k x}, \quad j(t, x)=\sum_{k \in \mathbb{Z}} j_{k}(t) e^{i k x},
$$

leads to real ODE systems for $U_{k}=\left(\begin{array}{c}u_{k} \\ v_{k}\end{array}\right):=\left(\begin{array}{c}\operatorname{Re}\left(\rho_{k}\right) \\ \operatorname{Im}\left(j_{k}\right)\end{array}\right)$ and $\widetilde{U}_{k}:=\left(\begin{array}{c}\operatorname{Im}\left(\rho_{k}\right) \\ \operatorname{Re}\left(j_{k}\right)\end{array}\right)$ :

$$
\frac{\mathrm{d}}{\mathrm{d} t} U_{k}+\mathrm{T}_{k} U_{k}=\mathrm{L} U_{k}
$$

where the skew symmetric matrix $\mathrm{T}_{k}:=\left(\begin{array}{cc}0 & -k \\ k & 0\end{array}\right)$ represents the transport operator, $\mathrm{L}:=\left(\begin{array}{cc}0 & 0 \\ 0 & -1\end{array}\right)$ represents the collision operator acting only on the microscopic component $j$, and $\widetilde{U}_{k}$ solves an analogous system with $\mathrm{T}_{k}$ replaced by $-\mathrm{T}_{k}$. Equation (1.7) is linear, and it is elementary to check that the eigenvalues of $\mathrm{L}-\mathrm{T}$ are given by $\lambda_{0, \pm}:=0,-1$ and $\lambda_{k, \pm}:=\left(-1 \pm i \sqrt{4 k^{2}-1}\right) / 2$ if $k \neq 0$. All solutions converge to an eigenstate of the zero eigenvalue: $U_{0}=\left(\rho_{0}, 0\right)$ and $U_{k}=0$ for $k \neq 0$. The convergence is exponential with its speed determined by the spectral gap $1 / 2$.

For $k \neq 0$, we can compute the entropy dissipation as

$$
\frac{\mathrm{d}}{\mathrm{d} t}\left(\frac{1}{2}\left|U_{k}(t)\right|^{2}\right)=-\left|v_{k}(t)\right|^{2},
$$

so that it is clear that no exponential decay directly follows, since the right hand side is not coercive and there is an unbounded increasing sequence $\left(t_{n}\right)_{n \in \mathbb{N}}$ such that $v_{k}\left(t_{n}\right) \rightarrow 0$. Note that microscopic coercivity holds with $\lambda_{m}=1$.

With $\Pi=\left(\begin{array}{ll}1 & 0 \\ 0 & 0\end{array}\right)$ and $\mathrm{J}=-\mathrm{Id}$, we find that $(\mathrm{T} \Pi)^{*} \mathrm{~J}(\mathrm{~T} \Pi)=\left(\begin{array}{cc}-k^{2} & 0 \\ 0 & 0\end{array}\right)$, thus giving for the macroscopic diffusion limit $\mathrm{d} u_{k}^{0} / \mathrm{d} t=-k^{2} u_{k}^{0}$, and showing also that 
macroscopic coercivity holds with $\lambda_{M}=1$. According to the strategy of Section 1.3 , for $k \neq 0$ we introduce the modified entropies

$$
\mathrm{H}_{k}(t)=\frac{1}{2}\left|U_{k}(t)\right|^{2}+\varepsilon \frac{k}{1+k^{2}} u_{k}(t) v_{k}(t), \quad t \geq 0 .
$$

Let us point out how this simple example highlights in the latter equation the surprising link with the theory of compensating functions of Kawashima; see 24] and [14, Section 3.11].

Observing that, for $k \geq 1$,

$$
\begin{aligned}
& \frac{1}{2}\left(1-\varepsilon \frac{k}{1+k^{2}}\right)\left|U_{k}\right|^{2} \leq \frac{1}{2}\left(1-\varepsilon \frac{k}{1+k^{2}}\right)\left|U_{k}\right|^{2}+\frac{\varepsilon}{2} \frac{k}{1+k^{2}}\left|u_{k}+v_{k}\right|^{2}=\mathrm{H}_{k} \\
& \mathrm{H}_{k}=\frac{1}{2}\left(1+\varepsilon \frac{k}{1+k^{2}}\right)\left|U_{k}\right|^{2}-\frac{\varepsilon}{2} \frac{k}{1+k^{2}}\left|u_{k}-v_{k}\right|^{2} \leq \frac{1}{2}\left(1+\varepsilon \frac{k}{1+k^{2}}\right)\left|U_{k}\right|^{2}
\end{aligned}
$$

using $\sup _{k \geq 1} \frac{k}{1+k^{2}}=\frac{1}{2} \leq 1$, and performing a similar computation for $k \leq-1$, we finally get

$$
\frac{1}{2}(1-\varepsilon)\left|U_{k}\right|^{2} \leq \mathrm{H}_{k} \leq \frac{1}{2}(1+\varepsilon)\left|U_{k}\right|^{2} .
$$

Hence, for any $\varepsilon \in(0,1), \mathrm{H}_{k}(t)$ decays exponentially if and only if $\left|U_{k}(t)\right|^{2}$ decays exponentially as well. Obviously, we have

$$
\frac{1}{2} \leq \frac{k^{2}}{1+k^{2}} \leq 1
$$

which makes it easy to compare $\mathrm{H}_{k}$ with $\frac{\mathrm{d}}{\mathrm{d} t} \mathrm{H}_{k}$, given by

$$
\begin{aligned}
\frac{\mathrm{d}}{\mathrm{d} t} \mathrm{H}_{k} & =-\varepsilon \frac{k^{2}}{1+k^{2}} u_{k}^{2}-\left(1-\varepsilon \frac{k^{2}}{1+k^{2}}\right) v_{k}^{2}-\varepsilon \frac{k}{1+k^{2}} u_{k} v_{k} \\
& \leq-\frac{\varepsilon}{2} u_{k}^{2}-(1-\varepsilon) v_{k}^{2}+\frac{\varepsilon}{2}\left|u_{k}\right|\left|v_{k}\right| \leq-\frac{\varepsilon}{2}\left(1-\lambda^{2}\right) u_{k}^{2}-\left(1-\varepsilon-\frac{\varepsilon}{8 \lambda^{2}}\right) v_{k}^{2}
\end{aligned}
$$

for any $\lambda \in(0,1)$. If $\varepsilon \in\left(0, \frac{8 \lambda^{2}}{8 \lambda^{2}+1}\right)$, the coercivity constant

$$
\kappa:=\min \left\{\frac{\varepsilon}{2}\left(1-\lambda^{2}\right), 1-\varepsilon-\frac{\varepsilon}{8 \lambda^{2}}\right\}
$$

is positive and

$$
\frac{\mathrm{d}}{\mathrm{d} t} \mathrm{H}_{k} \leq-\kappa\left|U_{k}\right|^{2} \leq-\frac{2 \kappa}{1+\varepsilon} \mathrm{H}_{k}
$$

This implies $\left|U_{k}(t)\right|$ decays like $e^{-\kappa t /(1+\varepsilon)}$. We may observe that

$$
\frac{\kappa}{1+\varepsilon}<\frac{\min \left\{\frac{\varepsilon}{2}, 1-\varepsilon\right\}}{1+\varepsilon} \leq \frac{1}{5},
$$

thus showing that the method is not optimal, in the sense that it does not give the exact decay rate, $1 / 2$, even when refining the above estimates and computing $\kappa$ for each $k$. 
1.5. Application to kinetic equations. Let us apply the abstract procedure of Section 1.3 to the setting of Section 1.1. Thus, we set

$$
\mathrm{\top} f:=v \cdot \nabla_{x} f-\nabla_{x} V \cdot \nabla_{v} f, \quad \Pi f:=\rho_{f} \frac{F}{\rho_{F}},
$$

where the potential $V$ is given as well as the energy profile $\Gamma$. We recall that the unique global equilibrium is $F(x, v)=\Gamma\left(\left|v^{2}\right| / 2+V(x)\right), x, v \in \mathbb{R}^{d}$. For such an equilibrium distribution, define the velocity moments up to the fourth order by

$$
\rho_{F}:=\int_{\mathbb{R}^{d}} F \mathrm{~d} v, \quad m_{F}:=\frac{1}{d} \int_{\mathbb{R}^{d}}|v|^{2} F \mathrm{~d} v, \quad M_{F}:=\int_{\mathbb{R}^{d}}|v|^{4} F \mathrm{~d} v
$$

and assume that they are measurable functions of $x$. We consider the Hilbert space $\mathcal{H}=\left\{f \in L^{2}(\mathrm{~d} \mu): \iint_{\mathbb{R}^{d} \times \mathbb{R}^{d}} f \mathrm{~d} v \mathrm{~d} x=0\right\}$, with $\mathrm{d} \mu(x, v)=\mathrm{d} x \mathrm{~d} v / F(x, v)$. The collision operator $\mathrm{L}$ remains unspecified at the moment, so that we shall defer the discussion of the microscopic coercivity for a while. A simple computation with $u=\rho_{f} / \rho_{F}$ shows that the macroscopic coercivity assumption is equivalent to a weighted Poincaré inequality:

Lemma 3. Assumption (H2) holds if and only if

$$
\int_{\mathbb{R}^{d}}\left|\nabla_{x} u\right|^{2} m_{F} \mathrm{~d} x \geq \lambda_{M} \int_{\mathbb{R}^{d}} u^{2} \rho_{F} \mathrm{~d} x
$$

for any $u \in L^{2}\left(\rho_{F} \mathrm{~d} x\right)$ with $\nabla_{x} u \in L^{2}\left(m_{F} \mathrm{~d} x\right)$ such that $\int_{\mathbb{R}^{d}} u \rho_{F} \mathrm{~d} x=0$.

In case of kinetic equations, Assumption (H3) is a consequence of the computation

$$
\mathrm{\top} \Pi f=F v \cdot \nabla_{x} u_{f},
$$

with $u_{f}:=\rho_{f} / \rho_{F}$, and of the observation that the right hand side is an odd function of $v$, whose mean value is zero. In other words: The macroscopic flux of the equilibrium distributions vanishes.

Concerning Assumption (H4), we remark that boundedness of $A L$ is possible even for unbounded collision operators $L$ (see Section 2). The boundedness assumption on $\mathrm{AT}(1-\Pi)$ can be interpreted as an elliptic regularity result for:

$$
u-\frac{1}{\rho_{F}} \nabla_{x} \cdot\left(m_{F} \nabla_{x} u\right)=w .
$$

Lemma 4. If there exists a positive constant $C$ such that

$$
\left\|\nabla_{x}^{2} u\right\|_{L^{2}\left(M_{F} \mathrm{~d} x\right)} \leq C\|w\|_{L^{2}\left(\rho_{F} \mathrm{~d} x\right)}
$$

for any $w \in L^{2}\left(\rho_{F} \mathrm{~d} x\right)$ and for any solution $u \in L^{2}\left(\rho_{F} \mathrm{~d} x\right)$ with $\nabla_{x} u \in L^{2}\left(m_{F} \mathrm{~d} x\right)$, then the operator $\mathrm{AT}(1-\Pi)$ is bounded on $\mathcal{H}$.

Proof. The operator A T $(1-\Pi)$ is bounded if and only if its adjoint

$$
[\mathrm{A} \mathrm{T}(1-\Pi)]^{*}=-(1-\Pi) \mathrm{T}^{2} \Pi\left[1+(\mathrm{T} \Pi)^{*}(\mathrm{~T} \Pi)\right]^{-1}
$$

is bounded. If $g=\left[1+(\mathrm{T} \Pi)^{*}(\mathrm{~T} \Pi)\right]^{-1} f$, then

$$
[\mathrm{A} \mathrm{T}(1-\Pi)]^{*} f=-(1-\Pi) \mathrm{T}^{2} \Pi g \quad \text { with } \quad g+(\mathrm{T} \Pi)^{*}(\mathrm{~T} \Pi) g=f,
$$

where the latter implies (1.9) for $u=u_{g}=\rho_{g} / \rho_{F}$ and $w=u_{f}=\rho_{f} / \rho_{F}$. Then

$$
\mathrm{T}^{2} \Pi g=F v \cdot \nabla_{x}\left(v \cdot \nabla_{x} u\right)+F \nabla_{x} V \cdot \nabla_{x} u
$$

results in

$$
[\mathrm{A} \mathrm{T}(1-\Pi)]^{*} f=-(1-\Pi)\left[F v \cdot \nabla_{x}\left(v \cdot \nabla_{x} u\right)\right] .
$$


This implies that for some positive constant $C$, we have

$$
\left\|[\mathrm{AT}(1-\Pi)]^{*} f\right\| \leq\left\|F v \cdot \nabla_{x}\left(v \cdot \nabla_{x} u\right)\right\| \leq c\left\|\nabla_{x}^{2} u\right\|_{L^{2}\left(M_{F} \mathrm{~d} x\right)},
$$

which completes the proof using $\left\|u_{f}\right\|_{L^{2}\left(\rho_{F} \mathrm{~d} x\right)}=\|\Pi f\|$.

\section{A FRAMEWORK FOR THE ELLIPTIC REgUlarity ESTIMATE}

Our goal is to give conditions on $V$ which are sufficient to establish the existence of a positive constant $C$ as in Lemma 4. In the applications considered below, the combination of weights $M_{F} \rho_{F} / m_{F}^{2}$ is constant. This motivates the notation

$$
w_{0}^{2}:=\rho_{F}, \quad w_{i}^{2}:=\left(\frac{m_{F}}{\rho_{F}}\right)^{i} w_{0}^{2} \quad \text { with } \quad i=1,2, \quad\|u\|_{i}:=\left\|u w_{i}\right\|_{L^{2}\left(\mathbb{R}^{d}\right)} .
$$

With $\rho=u \rho_{F}$, the Poincaré inequality in (H3) can then be rewritten as

$$
\left\|\nabla_{x} u\right\|_{1}^{2} \geq \lambda_{M}\|u\|_{0}^{2}
$$

under the zero average condition $\int_{\mathbb{R}^{d}} u \rho_{F} \mathrm{~d} x=0$, and the desired estimate (1.10) is

$$
\left\|\nabla_{x}^{2} u\right\|_{2} \leq C\left\|u_{f}\right\|_{0}
$$

for the solution of

$$
w_{0}^{2} u-\nabla_{x} \cdot\left(w_{1}^{2} \nabla_{x} u\right)=w_{0}^{2} u_{f} .
$$

Roughly speaking we just have to prove $\left(L^{2} \rightarrow H^{2}\right)$-regularization for a second order elliptic equation. However, different norms have to be taken into account. The result can only be shown under certain assumptions on the weights, which will later be translated into assumptions on the confining potential:

$$
\exists c_{1}>0, c_{2} \in[0,1) \text { such that }-w_{1}^{2} \Delta_{x}\left(\log w_{1}\right) \leq c_{1} w_{0}^{2}+c_{2}\left|\nabla_{x} w_{1}\right|^{2},
$$

$\exists c_{3}>0$ such that $\frac{w_{1}}{w_{0}}\left|\nabla_{x} W\right| \leq c_{3}\left(1+\frac{\left|\nabla_{x} w_{1}\right|}{w_{0}}\right)$ with $W:=\sqrt{1+\left|\frac{\nabla_{x} w_{1}}{w_{0}}\right|^{2}}$,

$$
\begin{gathered}
\exists c_{4}>0 \text { such that }\left|\nabla_{x}\left(\frac{w_{1}}{w_{0}}\right)\right| \leq c_{4} \frac{\left|\nabla_{x} w_{1}\right|}{w_{0}}, \\
\|W\|_{0}^{2}=\int_{\mathbb{R}^{d}} W^{2} \rho_{F} \mathrm{~d} x<\infty .
\end{gathered}
$$

Note that a condition on the third weight function $w_{2}$ could be deduced from (2.4)(2.5)-(2.6) since any two of the weight functions determine the last one. The goal of this section is to prove the following $H^{2}$-regularity estimate.

Proposition 5. Let (2.1), (2.4), (2.5), (2.6), and (2.7) hold. Then the solution u of (2.3) satisfies (2.2).

By Lemma 4, this shows that the operator A T $(1-\Pi)$ is bounded. 
2.1. Improved Poincaré inequalities. We start with an improvement of the Poincaré inequality (2.1).

Lemma 6. Let (2.1) and (2.4) hold. There exists $\kappa>0$ such that

$$
\left\|\nabla_{x} u\right\|_{1}^{2} \geq \kappa\left\|u \frac{\nabla_{x} w_{1}}{w_{0}}\right\|_{0}^{2} \quad \text { for any } u \in L^{2}\left(\rho_{F} \mathrm{~d} x\right) \text { with } \int_{\mathbb{R}^{d}} u \rho_{F} \mathrm{~d} x=0 .
$$

Proof. With the identity $w_{1} \nabla_{x} u=\nabla_{x}\left(w_{1} u\right)-u \nabla_{x} w_{1}$, the inequality

$$
\begin{aligned}
\left\|\nabla_{x} u\right\|_{1}^{2} & \geq \int_{\mathbb{R}^{d}} u^{2}\left|\nabla_{x} w_{1}\right|^{2} \mathrm{~d} x-2 \int_{\mathbb{R}^{d}} u \nabla_{x} w_{1} \cdot \nabla_{x}\left(u w_{1}\right) \mathrm{d} x \\
& =\left\|u \frac{\nabla_{x} w_{1}}{w_{0}}\right\|_{0}^{2}+\int_{\mathbb{R}^{d}} u^{2} w_{1}^{2} \Delta_{x}\left(\log w_{1}\right) \mathrm{d} x
\end{aligned}
$$

is easily derived. Now (2.1) and (2.4) imply

$$
\left\|\nabla_{x} u\right\|_{1}^{2} \geq\left(1-c_{2}\right)\left\|u \frac{\nabla_{x} w_{1}}{w_{0}}\right\|_{0}^{2}-\frac{c_{1}}{\lambda_{M}}\left\|\nabla_{x} u\right\|_{1}^{2} .
$$

This completes the proof with $\kappa=\lambda_{M}\left(1-c_{2}\right) /\left(\lambda_{M}+c_{1}\right)$.

Lemma 7. Let (2.1), (2.4), (2.5), and (2.7) hold. There exists $\kappa^{\prime}>0$ such that

$$
\left\|W \nabla_{x} u\right\|_{1}^{2} \geq \kappa^{\prime}\left\|W u \frac{\nabla_{x} w_{1}}{w_{0}}\right\|_{0}^{2} \quad \text { for any } u \in L^{2}\left(\rho_{F} \mathrm{~d} x\right) \text { with } \int_{\mathbb{R}^{d}} u \rho_{F} \mathrm{~d} x=0 .
$$

Proof. We apply (2.8) with $u$ replaced by $(u W-\bar{u})$, where $\bar{u}:=\int_{\mathbb{R}^{d}} u W \rho_{F} \mathrm{~d} x$. We recall that $\int_{\mathbb{R}^{d}} \rho_{F} \mathrm{~d} x=\iint_{\mathbb{R}^{d} \times \mathbb{R}^{d}} F \mathrm{~d} v \mathrm{~d} x=1$. We thus obtain

$$
\kappa\|(u W-\bar{u}) W\|_{0}^{2} \leq\left\|\nabla_{x}(W u)\right\|_{1}^{2} .
$$

By expanding the left hand side, we get

$$
\kappa\left\|W^{2} u\right\|_{0}^{2} \leq\left\|\nabla_{x}(W u)\right\|_{1}^{2}+2 \kappa\left(\int_{\mathbb{R}^{d}} W^{3} u w_{0}^{2} \mathrm{~d} x\right)\|W\|_{0}\|u\|_{0} .
$$

Using

$$
\int_{\mathbb{R}^{d}} W^{2} u W \rho_{F} \mathrm{~d} x \leq \frac{1}{2 a}\left\|W^{2} u\right\|_{0}^{2}+\frac{a}{2}\|W\|_{0}^{2}
$$

with $a:=2\|W\|_{0}\|u\|_{0}$, we obtain

$$
\begin{aligned}
2 \kappa \int_{\mathbb{R}^{d}} W^{3} u w_{0}^{2} \mathrm{~d} x\|W\|_{0}\|u\|_{0} \leq & \kappa a\left(\frac{1}{2 a}\left\|W^{2} u\right\|_{0}^{2}+\frac{a}{2}\|W\|_{0}^{2}\right) \\
& =\frac{\kappa}{2}\left\|W^{2} u\right\|_{0}^{2}+2 \kappa\|W\|_{0}^{4}\|u\|_{0}^{2} .
\end{aligned}
$$

On the other hand, we can also expand the square in $\left\|\nabla_{x}(W u)\right\|_{1}^{2}$ :

and by (2.5), get

$$
\left\|\nabla_{x}(W u)\right\|_{1}^{2} \leq 2\left\|W \nabla_{x} u\right\|_{1}^{2}+2\left\|u \nabla_{x} W \frac{w_{1}}{w_{0}}\right\|_{0}^{2},
$$

$$
\left\|u \nabla_{x} W \frac{w_{1}}{w_{0}}\right\|_{0}^{2} \leq c_{3}^{2}\left\|u\left(1+\frac{\left|\nabla_{x} w_{1}\right|}{w_{0}}\right)\right\|_{0}^{2} \leq 2 c_{3}^{2}\|W u\|_{0}^{2} .
$$

Collecting all terms, we finally end up with

$$
\kappa\left\|W^{2} u\right\|_{0}^{2} \leq 2\left\|W \nabla_{x} u\right\|_{1}^{2}+4 c_{3}^{2}\|W u\|_{0}^{2}+\frac{\kappa}{2}\left\|W^{2} u\right\|_{0}^{2}+2 \kappa\|W\|_{0}^{4}\|u\|_{0}^{2},
$$


which, using (2.1), (2.8), and $W \geq 1$ establishes the inequality

$$
\frac{\kappa}{2}\left\|W^{2} u\right\|_{0}^{2} \leq\left(2+4 c_{3}^{2}\left(\frac{1}{\kappa}+\frac{1}{\kappa}\right)+2 \frac{\kappa}{\lambda_{M}}\|W\|_{0}^{4}\right)\left\|W \nabla_{x} u\right\|_{1}^{2} .
$$

2.2. The regularity estimate. Now we start working on equation (2.3). The standard energy estimate gives

$$
\|u\|_{0}^{2}+\left\|\nabla_{x} u\right\|_{1}^{2} \leq\left\|u_{f}\right\|_{0}^{2} .
$$

With $W=\sqrt{1+\left|\nabla_{x} w_{1}\right|^{2} / w_{0}^{2}}$, Lemma 6 leads to the improved $L^{2}$-estimate

$$
\|u W\|_{0} \leq \frac{1}{\kappa}\left\|u_{f}\right\|_{0} .
$$

Lemma 6 can also be used to get an improved $H^{1}$-estimate.

Lemma 8. Let (2.1), (2.4), (2.5), and (2.7) hold. Then any solution of (2.3) such that $\int_{\mathbb{R}^{d}} u \rho_{F} \mathrm{~d} x=0$ satisfies

$$
\left\|W \nabla_{x} u\right\|_{1} \leq C\left\|u_{f}\right\|_{0} .
$$

Proof. Multiplication of (2.3) by $u W^{2}$ and integration gives

$$
\|u W\|_{0}^{2}+\left\|W \nabla_{x} u\right\|_{1}^{2} \leq\left\|u W^{2}\right\|_{0}\left\|u_{f}\right\|_{0}-\int_{\mathbb{R}^{d}} w_{1}^{2} u \nabla_{x} u \cdot \nabla_{x}\left(W^{2}\right) \mathrm{d} x .
$$

Since $W^{2} \leq 1+W\left|\nabla_{x} w_{1}\right| / w_{0}$, (2.1) and Lemma 7 imply

$$
\left\|u W^{2}\right\|_{0}^{2} \leq 2\left(\frac{1}{\lambda_{M}}+\frac{1}{\kappa^{\prime}}\right)\left\|W \nabla_{x} u\right\|_{1}^{2} .
$$

The integrand in the last term above can be estimated by

$$
w_{0}|u| w_{1}\left|\nabla_{x} u\right| \frac{2 w_{1} W\left|\nabla_{x} W\right|}{w_{0}} \leq 2 \sqrt{2} c_{3} w_{0}|u| w_{1}\left|\nabla_{x} u\right| W^{2}
$$

with the help of (2.5), so that the integral is bounded by

$$
2 \sqrt{2} c_{3}\|u W\|_{0}\left\|W \nabla_{x} u\right\|_{1} \leq 2 \sqrt{2} \frac{c_{3}}{\kappa}\left\|u_{f}\right\|_{0}\left\|W \nabla_{x} u\right\|_{1} .
$$

Combining our results gives

$$
\left\|W \nabla_{x} u\right\|_{1}^{2} \leq C\left\|u_{f}\right\|_{0}\left\|W \nabla_{x} u\right\|_{1}
$$

with $C=\sqrt{2\left(1 / \lambda_{M}+1 / \kappa^{\prime}\right)}+2 \sqrt{2} \frac{c_{3}}{\kappa}$, thus completing the proof.

Proof of Proposition 5, We follow the standard procedure for proving $H^{2}$-regularity of the solutions of second order elliptic equations with $L^{2}$ right hand sides: multiply (2.3) with $\nabla_{x} \cdot\left(\nabla_{x} u w_{1}^{2} / w_{0}^{2}\right)$ and integrate by parts twice. We also use the consequence $w_{2}=w_{1}^{2} / w_{0}$ of the relations between the weights:

$$
\begin{array}{r}
\left\|\nabla_{x}^{2} u\right\|_{2}^{2}=\int_{\mathbb{R}^{d}} w_{0}\left(u-u_{f}\right) w_{2} \Delta_{x} u \mathrm{~d} x+\int_{\mathbb{R}^{d}} w_{0}^{2}\left(u-u_{f}\right) \nabla_{x} u \cdot \nabla_{x}\left(\frac{w_{1}^{2}}{w_{0}^{2}}\right) \mathrm{d} x \\
-\int_{\mathbb{R}^{d}} w_{1}^{2} \nabla_{x} u \nabla_{x}^{2} u \nabla_{x}\left(\frac{w_{1}^{2}}{w_{0}^{2}}\right) \mathrm{d} x-\int_{\mathbb{R}^{d}} \frac{w_{1}^{2}}{w_{0}^{2}} \nabla_{x} u \nabla_{x}^{2} u \nabla_{x}\left(w_{1}^{2}\right) \mathrm{d} x \\
-\int_{\mathbb{R}^{d}}\left[\nabla_{x} u \cdot \nabla_{x}\left(\frac{w_{1}^{2}}{w_{0}^{2}}\right)\right]\left[\nabla_{x} u \cdot \nabla_{x}\left(w_{1}^{2}\right)\right] \mathrm{d} x \\
=I_{1}+I_{2}+I_{3}+I_{4}+I_{5} .
\end{array}
$$


The first integral is easily estimated:

$$
\left|I_{1}\right| \leq\left(\|u\|_{0}+\left\|u_{f}\right\|_{0}\right)\left\|\nabla_{x}^{2} u\right\|_{2} \leq 2\left\|u_{f}\right\|_{0}\left\|\nabla_{x}^{2} u\right\|_{2},
$$

by (2.9). For the second integral we use (2.6) and Lemma 8

$$
\begin{aligned}
\left|I_{2}\right| & \leq 2 c_{4} \int_{\mathbb{R}^{d}} w_{0}\left(u-u_{f}\right) w_{1}\left|\nabla_{x} u\right| \frac{\left|\nabla_{x} w_{1}\right|}{w_{0}} \mathrm{~d} x \\
& \leq 2 c_{4}\left\|u-u_{f}\right\|_{0}\left\|W \nabla_{x} u\right\|_{1} \leq 4 c_{4} C\left\|u_{f}\right\|_{0}^{2} .
\end{aligned}
$$

With the third, fourth, and fifth integrals we proceed similarly:

$$
\begin{aligned}
\left|I_{3}\right| & \leq 2 c_{4} \int_{\mathbb{R}^{d}} w_{1}\left|\nabla_{x} u\right| w_{2}\left|\nabla_{x}^{2} u\right| \frac{\left|\nabla_{x} w_{1}\right|}{w_{0}} \mathrm{~d} x \\
\leq & 2 c_{4}\left\|W \nabla_{x} u\right\|_{1}\left\|\nabla_{x}^{2} u\right\|_{2} \leq 2 c_{4} C\left\|u_{f}\right\|_{0}\left\|\nabla_{x}^{2} u\right\|_{2}, \\
\left|I_{4}\right| \leq & 2 \int_{\mathbb{R}^{d}} w_{1}\left|\nabla_{x} u\right| w_{2}\left|\nabla_{x}^{2} u\right| \frac{\left|\nabla_{x} w_{1}\right|}{w_{0}} \mathrm{~d} x \leq 2 c_{4} C\left\|u_{f}\right\|_{0}\left\|\nabla_{x}^{2} u\right\|_{2}, \\
\left|I_{5}\right| \leq & 4 \int_{\mathbb{R}^{d}} w_{1}^{2}\left|\nabla_{x} u\right|^{2}\left|\nabla_{x}\left(\frac{w_{1}}{w_{0}}\right)\right| \frac{\left|\nabla_{x} w_{1}\right|}{w_{0}} \mathrm{~d} x \\
& \leq 4 c_{4} \int_{\mathbb{R}^{d}} w_{1}^{2}\left|\nabla_{x} u\right|^{2} \frac{\left|\nabla_{x} w_{1}\right|^{2}}{w_{0}^{2}} \mathrm{~d} x \leq 4 c_{4}\left\|W \nabla_{x} u\right\|_{1}^{2} \leq 4 c_{4} C\left\|u_{f}\right\|_{0}^{2} .
\end{aligned}
$$

The combination of our results gives

$$
\left\|\nabla_{x}^{2} u\right\|_{2}^{2} \leq K\left\|u_{f}\right\|_{0}\left(\left\|u_{f}\right\|_{0}+\left\|\nabla_{x}^{2} u\right\|_{2}\right)
$$

for some explicit constant $K>0$, which completes the proof.

\section{MAXWELLIAN EQUILIBRIA}

When the local equilibrium is a Maxwellian distribution, the global equilibrium has the form

$$
F(x, v)=\rho_{F}(V(x)) \mathfrak{M}(v), \quad \text { with } \rho_{F}(V)=e^{-V} \quad \text { and } \quad \mathfrak{M}(v)=\frac{e^{-|v|^{2} / 2}}{(2 \pi)^{d / 2}}
$$

In this framework, Assumption (H0) is a consequence of

Assumption (H0.1): The external potential $V \in C^{2}\left(\mathbb{R}^{d}\right)$ is such that $e^{-V} \in$ $L^{1}(\mathrm{~d} x)$.

As far as the macroscopic coercivity condition (H2) and the boundedness of A T $(1-\Pi)$, i.e. the first part of $(\mathbf{H} 4)$, are concerned, no further details of the collision operator are required. Consider first the issue of finding equivalent conditions for (H2).

With $w:=\rho e^{V / 2}$ the macroscopic coercivity condition (1.8) is equivalent to

$$
\int_{\mathbb{R}^{d}}\left[\left|\nabla_{x} w\right|^{2}+\left(\frac{1}{4}\left|\nabla_{x} V\right|^{2}-\frac{1}{2} \Delta V\right) w^{2}\right] \mathrm{d} x \geq \lambda_{M} \int_{\mathbb{R}^{d}} w^{2} \mathrm{~d} x,
$$

under the orthogonality condition $\int_{\mathbb{R}^{d}} w e^{-V / 2} \mathrm{~d} x=0$. The first eigenvalue of the Schrödinger operator $\mathcal{H}:=-\Delta+\frac{1}{4}\left|\nabla_{x} V\right|^{2}-\frac{1}{2} \Delta V$ is zero. It is nondegenerate, and the corresponding eigenfunction is $w=e^{-V / 2}$. According to 30, inequality (1.8) holds if and only if the lower end of the continuous spectrum of $\mathcal{H}$ is positive, that is

Assumption (H2.1): $\quad \liminf |x| \rightarrow \infty\left(\left|\nabla_{x} V\right|^{2}-2 \Delta V\right)>0$. 
As a consequence, macroscopic coercivity holds if: $\Delta V$ is negligible compared to $\left|\nabla_{x} V\right|^{2}$ as $|x| \rightarrow \infty$, and $\liminf _{|x| \rightarrow \infty}\left|\nabla_{x} V\right|>0$. An example of such a potential is $V(x)=\left(1+|x|^{2}\right)^{\beta}$ for some $\beta \geq 1 / 2$. See for instance [35, A.19, Some criteria for Poincaré inequalities, pg. 137] for an elementary proof if $\lim _{|x| \rightarrow \infty}\left(\left|\nabla_{x} V\right|^{2}-2 \Delta V\right)$

$=\infty$, and [1] for some recent considerations on Poincaré inequalities when $e^{-V}$ is a probability measure.

Since all three weights $\rho_{F}, m_{F}$, and $M_{F}$ are constant multiples of $e^{-V}$, the framework of Section 2 can be used for the boundedness of A T (1- I). Assumptions (2.4), (2.5) are satisfied if

Assumption (H4.1): There exist constants $c_{1}>0, c_{2} \in[0,1)$, and $c_{3}>0$, such that

$$
\Delta_{x} V \leq c_{1}+\frac{c_{2}}{2}\left|\nabla_{x} V\right|^{2}, \quad\left|\nabla_{x}^{2} V\right| \leq c_{3}\left(1+\left|\nabla_{x} V\right|\right) .
$$

Assumption (2.6) holds trivially (since $w_{1} / w_{0}=$ constant), and (2.7) can be translated to

$$
\int_{\mathbb{R}^{d}}\left|\nabla_{x} V\right|^{2} e^{-V} \mathrm{~d} x<\infty
$$

which follows from (H0.1) and (H4.1) by

$$
\begin{aligned}
\int_{\mathbb{R}^{d}}\left|\nabla_{x} V\right|^{2} e^{-V} \mathrm{~d} x & =-\int_{\mathbb{R}^{d}} \nabla_{x} V \cdot \nabla_{x} e^{-V} \mathrm{~d} x=\int_{\mathbb{R}^{d}} \Delta_{x} V e^{-V} \mathrm{~d} x \\
& \leq c_{1} \int_{\mathbb{R}^{d}} e^{-V} \mathrm{~d} x+\frac{c_{2}}{2} \int_{\mathbb{R}^{d}}\left|\nabla_{x} V\right|^{2} e^{-V} \mathrm{~d} x .
\end{aligned}
$$

3.1. BGK operator. For the BGK collision operator

$$
\mathrm{L}=\Pi-1,
$$

the microscopic coercivity condition $(\mathbf{H 1})$ is trivially satisfied with $\lambda_{m}=1$, and, since $L$ is bounded (by 1 ), the boundedness of $A L$ follows from Lemma 1 .

Theorem 9. Let $\mathrm{L}=\Pi-1$, and let the external potential satisfy (H0.1), (H2.1), and (H4.1). Then solutions to (1.1) with initial data in $L^{2}(\mathrm{~d} \mu)$ decay exponentially to the global equilibrium given by (3.1).

This result is an improvement upon the work of Hérau [21, since the requirements for the external potential are weaker. In particular, Hérau's result requires potentials with at most quadratic growth at infinity, whereas an arbitrary superlinear growth is permitted by $(\mathbf{H 2 . 1}),(\mathbf{H} 4.1)$.

3.2. Fokker-Planck operator. For the Fokker-Planck collision operator

$$
\mathrm{L} f=\nabla_{v} \cdot\left(\nabla_{v} f+v f\right),
$$

the microscopic coercivity condition (H1) is equivalent to the Poincaré inequality for the Gaussian measure $\mathfrak{M}(v) \mathrm{d} v$, which satisfies

$$
\begin{aligned}
-\langle\mathrm{L} f, f\rangle & =\int_{\mathbb{R}^{d}} e^{V} \int_{\mathbb{R}^{d}}\left|\nabla_{v} \frac{(1-\Pi) f}{\mathfrak{M}}\right|^{2} \mathfrak{M}(v) \mathrm{d} v \mathrm{~d} x \\
& \geq \lambda_{m} \int_{\mathbb{R}^{d}} e^{V} \int_{\mathbb{R}^{d}}\left|\frac{(1-\Pi) f}{\mathfrak{M}}\right|^{2} \mathfrak{M}(v) \mathrm{d} v \mathrm{~d} x=\lambda_{m}\|(1-\Pi) f\|^{2} .
\end{aligned}
$$

A seemingly surprising fact is the boundedness of $A L$, although $L$ is an unbounded operator. Since $\mathrm{A}=-\left(1+(\mathrm{T} \Pi)^{*}(\mathrm{~T} \Pi)\right)^{-1} \Pi \mathrm{T}$ and $\Pi \mathrm{T} f=\frac{F}{\rho_{F}} \nabla_{x} \cdot j_{f}$, where $j_{f}$ is 
the flux given by $j_{f}=\int_{\mathbb{R}^{d}} v f \mathrm{~d} v$, the identity $j_{\mathrm{L} f}=-j_{f}$ implies $\mathrm{AL}=-\mathrm{A}$, and the boundedness of $A L$ is a consequence of Lemma 1. In fact this boundedness follows from the strong regularizing property of the projection $\Pi$ in the velocity variable $v$.

Theorem 10. Let $\mathrm{L} f=\nabla_{v} \cdot\left(\nabla_{v} f+v f\right)$ and assume that the external potential satisfies (H0.1), (H2.1), and (H4.1). Then solutions to (1.1) with initial data in $L^{2}(\mathrm{~d} \mu)$ decay exponentially to the global equilibrium given by (3.1).

The above assumptions are similar to those of [35], which are weaker than those of [22]. Moreover this result is an important improvement compared to [35] as it involves an $L^{2}$ setting rather than an $H^{1}$ setting. Let us emphasize that the latter point is not a technical issue and answers an open question raised in [35, Part II, Section 13]).

3.3. Scattering operators (without detailed balance). Consider a scattering operator that can be written as

$$
(\mathrm{L} f)(v)=\int_{\mathbb{R}^{d}}\left[k\left(v^{*} \rightarrow v\right) f\left(v^{*}\right)-k\left(v \rightarrow v^{*}\right) f(v)\right] \mathrm{d} v^{*}, \quad k \geq 0,
$$

where $k\left(v^{*} \rightarrow v\right)$ denotes the transition probability of changing the velocity $v^{*}$ into $v$. Such an equation obviously conserves mass. Rotational symmetry can be enforced as a consequence of the assumption $k\left(R v^{*} \rightarrow R v\right)=k\left(v^{*} \rightarrow v\right)$, for all $v, v^{*}$, and for all rotation matrices $R$. Detailed balance would mean that the integrand vanishes pointwise, whenever $f$ is a local equilibrium distribution. We shall only require that $\mathrm{L} \mathfrak{M}=0$, i.e., the global balance. In the right hand side $f$ can be replaced by $(1-\Pi) f$. It has been shown in [7] that in this case an H-theorem holds:

$$
-\int_{\mathbb{R}^{d}} \mathrm{~L} f \frac{f}{\mathfrak{M}} \mathrm{d} v=\frac{1}{4} \int_{\mathbb{R}^{d}}\left(\frac{k\left(v^{*} \rightarrow v\right)}{\mathfrak{M}}+\frac{k\left(v \rightarrow v^{*}\right)}{\mathfrak{M}^{*}}\right) \mathfrak{M} \mathfrak{M}^{*}\left(\frac{f}{\mathfrak{M}}-\frac{f^{*}}{\mathfrak{M}^{*}}\right)^{2} \mathrm{~d} v^{*} \mathrm{~d} v,
$$

where $f^{*}$ denotes $f\left(v^{*}\right)$. A sufficient condition for microscopic coercivity is

Assumption (H1.1): $\quad \frac{k\left(v^{*} \rightarrow v\right)}{\mathfrak{M}}+\frac{k\left(v \rightarrow v^{*}\right)}{\mathfrak{M}^{*}} \geq 2 \lambda_{m}>0$.

Note that, because $\mathrm{L} \mathfrak{M}=0$, the collision frequency $\nu(v)=\int_{\mathbb{R}^{d}} k\left(v \rightarrow v^{*}\right) \mathrm{d} v^{*}$ can be written as

$$
\nu(v)=\frac{1}{2} \int_{\mathbb{R}^{d}}\left(\frac{k\left(v^{*} \rightarrow v\right)}{\mathfrak{M}}+\frac{k\left(v \rightarrow v^{*}\right)}{\mathfrak{M}^{*}}\right) \mathfrak{M}^{*} \mathrm{~d} v^{*} .
$$

Thus, (H1.1) implies $\nu(v) \geq \lambda_{m}$.

For proving the boundedness of $\mathrm{A} L$, note that $g=\mathrm{A} L f$ can be written as $g=u F$ with

$$
u e^{-V}-\nabla_{x} \cdot\left(e^{-V} \nabla_{x} u\right)=-\nabla_{x} \cdot j_{\mathrm{L} f} .
$$

Multiplication by $u$ and integration gives

$$
\int_{\mathbb{R}^{d}} u^{2} e^{-V} \mathrm{~d} x+\int_{\mathbb{R}^{d}}\left|\nabla_{x} u\right|^{2} e^{-V} \mathrm{~d} x=\int_{\mathbb{R}^{d}} \nabla_{x} u \cdot j_{\mathrm{L} f} \mathrm{~d} x .
$$


For the gain term $\mathrm{L}^{+} f=\int_{\mathbb{R}^{d}} k\left(v^{*} \rightarrow v\right) f^{*} \mathrm{~d} v^{*}$, by applying the Cauchy-Schwarz inequality twice, we get

$$
\begin{aligned}
\left|j_{\mathrm{L}+f}\right| & \leq \int_{\mathbb{R}^{d}}|v|\left[\int_{\mathbb{R}^{d}} \frac{\left|f^{*}\right|^{2}}{\mathfrak{M}^{*}} \mathrm{~d} v^{*} \int_{\mathbb{R}^{d}} k\left(v^{*} \rightarrow v\right)^{2} \mathfrak{M}^{*} \mathrm{~d} v^{*}\right]^{\frac{1}{2}} \mathrm{~d} v \\
& \leq\left[\int_{\mathbb{R}^{d}} \frac{\left|f^{*}\right|^{2}}{\mathfrak{M}^{*}} \mathrm{~d} v^{*} \iint_{\mathbb{R}^{d} \times \mathbb{R}^{d}}|v|^{2} k\left(v^{*} \rightarrow v\right)^{2} \frac{\mathfrak{M}^{*}}{\mathfrak{M}} \mathrm{d} v^{*} \mathrm{~d} v\right]^{\frac{1}{2}} .
\end{aligned}
$$

An analogous estimate for the loss term holds, so that we finally have

$$
\left|\int_{\mathbb{R}^{d}} \nabla_{x} u \cdot j_{\mathrm{L} f} \mathrm{~d} x\right| \leq C\left(\int_{\mathbb{R}^{d}}\left|\nabla_{x} u\right|^{2} e^{-V} \mathrm{~d} x\right)^{1 / 2}\|f\|
$$

under the assumption

$$
\text { Assumption (H4.2): } \quad \iint_{\mathbb{R}^{d} \times \mathbb{R}^{d}}\left(|v|^{2}+\left|v^{*}\right|^{2}\right) k\left(v^{*} \rightarrow v\right)^{2} \frac{\mathfrak{M}^{*}}{\mathfrak{M}} \mathrm{d} v^{*} \mathrm{~d} v<\infty \text {. }
$$

As a consequence,

$$
\|\mathrm{A} L f\|=\left(\int_{\mathbb{R}^{d}}|u|^{2} e^{-V} \mathrm{~d} x\right)^{1 / 2} \leq C\|f\|
$$

and $\mathrm{AL}$ is bounded. Combined with (H4.1), (H4.2) shows that (H4) holds.

Theorem 11. Let $\mathrm{L}$ be given by (3.2). If (H0.1), (H1.1), (H2.1), (H4.1), and (H4.2) hold, then solutions to (1.1) with initial data in $L^{2}(\mathrm{~d} \mu)$ decay exponentially to the global equilibrium given by (3.1).

\section{Linearized BGK operators}

4.1. Motivation: Nonlinear models. Our motivation in this section comes from nonlinear BGK models with collision operators of the form

$$
Q(f)=\gamma\left(\frac{1}{2}|v|^{2}-\bar{\mu}\left(\rho_{f}\right)\right)-f .
$$

The operator is determined by the energy profile $\gamma(E) \geq 0$ which is assumed to be monotone decaying on $\gamma^{-1}(0, \infty)$. The (strictly increasing) function $\bar{\mu}(\rho)$ is defined implicitly by the requirement of local mass conservation, i.e.

$$
\int_{\mathbb{R}^{d}} \gamma\left(\frac{1}{2}|v|^{2}-\bar{\mu}(\rho)\right) \mathrm{d} v=\rho .
$$

Global equilibria of the nonlinear equation

$$
\partial_{t} f+\mathrm{T} f=Q(f)
$$

are given by

$$
f_{\infty}(x, v)=\gamma\left(E(x, v)-\mu_{\infty}\right), \quad E(x, v)=\frac{|v|^{2}}{2}+V(x),
$$

where the constant $\mu_{\infty}$ is determined by the total mass, and the macroscopic equilibrium density $\rho_{\infty}$ is determined through the equation $\mu_{\infty}-V(x)=\bar{\mu}\left(\rho_{\infty}(x)\right)$. In this section, we shall investigate the linearized stability of these equilibria, leading to the linear equation (1.1) with the linearized collision operator $L=\Pi-1$ with

$$
F(x, v)=-\gamma^{\prime}\left(E(x, v)-\mu_{\infty}\right), \quad \rho_{F}(x)=\frac{1}{\bar{\mu}^{\prime}\left(\rho_{\infty}(x)\right)} .
$$


Note that in the Maxwellian case $\gamma(E)=e^{-E}, \bar{\mu}(\rho)=\log \left(\rho(2 \pi)^{-d / 2}\right)$, the operator $Q$ is linear, and therefore equal to $\mathrm{L}$. This case has already been investigated in Section 3.1

The macroscopic limit

$$
\partial_{t} \rho=\nabla_{x} \cdot\left(\nabla_{x} \nu(\rho)+\rho \nabla_{x} V\right)
$$

of the nonlinear equation is a drift-diffusion equation with nonlinear diffusivity $\sigma(\rho)=\nu^{\prime}(\rho)=\rho \bar{\mu}^{\prime}(\rho)$ (see 11] for a justification). Macroscopic limit and linearization commute in the sense that the linearization

$$
\partial_{t} \rho=\nabla_{x} \cdot\left(\nabla_{x}\left(\sigma\left(\rho_{\infty}\right) \rho\right)+\rho \nabla_{x} V\right)
$$

of the macroscopic equation is the macroscopic limit of the linearized kinetic equation.

In the following section we consider a family of equilibrium energy distributions $\gamma$, giving rise to nonlinear diffusions of fast diffusion type. As in Section 3.1. boundedness of $\mathrm{L}$ and microscopic coercivity are straightforward. Since $j_{\mathrm{L} f}=-j_{f}$, $A L$ is bounded, and $L A=0$ is easy to check. It remains to check the macroscopic coercivity condition and the boundedness of AT $(1-\Pi)$, corresponding to (H2) and (H4) respectively.

4.2. Fast diffusion. The choice $\gamma(E)=E^{-d / 2-1 /(1-m)}$ and $m<1$ leads to

$$
\nu(\rho)=c \begin{cases}\rho^{m} & \text { for } m \neq 0, \\ \log \rho & \text { for } m=0,\end{cases}
$$

with a constant $c>0$ depending on $m$ and $d$. For $m<1$, we also compute the moments

$$
\left\{\begin{array}{l}
\rho_{F}=c_{0}\left(V-\mu_{\infty}\right)^{-1-1 /(1-m)}, \\
m_{F}=c_{1}\left(V-\mu_{\infty}\right)^{-1 /(1-m)}, \\
M_{F}=c_{2}\left(V-\mu_{\infty}\right)^{1-1 /(1-m)},
\end{array}\right.
$$

where the positive constants $c_{0}, c_{1}, c_{2}$ depend on $m$ and $d$. For the external potential we shall, for notational convenience, only consider the choice

$$
V(x)-\mu_{\infty}=\left(1+|x|^{2}\right)^{\beta}, \quad \beta>0 .
$$

However, all our results easily extend to potentials whose asymptotic behaviour as $|x| \rightarrow \infty$ is given by (4.1). With these choices,

Assumption (H0.2): $\quad \beta>\frac{d(1-m)}{2(2-m)}$

is necessary and sufficient for $\rho_{F} \in L^{1}(\mathrm{~d} x)$.

Macroscopic coercivity is related to Hardy-Poincaré inequalities. In [2] 4, for any $d \geq 3, \alpha \neq \alpha^{*}:=-(d-2) / 2$, it is proved that there is an explicit positive constant $\mathcal{C}_{\alpha, d}$, such that

$$
\int_{\mathbb{R}^{d}}\left|\nabla_{x} u\right|^{2}\left(1+|x|^{2}\right)^{\alpha} \mathrm{d} x \geq \mathcal{C}_{\alpha, d} \int_{\mathbb{R}^{d}} u^{2}\left(1+|x|^{2}\right)^{\alpha-1} \mathrm{~d} x
$$

for all $u \in H^{1}\left(\left(1+|x|^{2}\right)^{\alpha} \mathrm{d} x\right)$, under the additional condition

$$
\int_{\mathbb{R}^{d}} u\left(1+|x|^{2}\right)^{\alpha-1} \mathrm{~d} x=0 \quad \text { if } \quad \alpha<\alpha^{*},
$$


in which case the measure $\left(1+|x|^{2}\right)^{\alpha-1} \mathrm{~d} x$ is bounded. The Hardy-Poincaré inequality is equivalent to macroscopic coercivity (H2) for $\beta=1$. The following small generalization is even more useful for our purposes.

Corollary 12. Let $d \geq 3, \alpha_{1} \geq \alpha_{2}+1$, and $\alpha_{1} \neq \alpha^{*}:=1-d / 2$ if $\alpha_{1}=\alpha_{2}+1$. Let $w$ be a function such that $0 \leq w(x) \leq c\left(1+|x|^{2}\right)^{\alpha_{2}}$ for any $x \in \mathbb{R}^{d}$, for some $c>0$. Then there exists a positive constant $\mathcal{K}_{\alpha_{1}, \alpha_{2}, d}$ such that

$$
\int_{\mathbb{R}^{d}}\left|\nabla_{x} u\right|^{2}\left(1+|x|^{2}\right)^{\alpha_{1}} \mathrm{~d} x \geq \mathcal{K}_{\alpha_{1}, \alpha_{2}, d} \int_{\mathbb{R}^{d}} u^{2} w \mathrm{~d} x
$$

for any $u$ such that $\int_{\mathbb{R}^{d}} u w \mathrm{~d} x=0$ if $\int_{\mathbb{R}^{d}} w \mathrm{~d} x<\infty$.

Proof. The assumptions on $\alpha_{1}$ and $\alpha_{2}$ allow us to choose $\alpha \neq \alpha^{*}$ with $\alpha_{2}+1 \leq$ $\alpha \leq \alpha_{1}$. Then Theorem 1 in [2] implies

$$
\begin{aligned}
& \int_{\mathbb{R}^{d}}\left|\nabla_{x} u\right|^{2}\left(1+|x|^{2}\right)^{\alpha_{1}} \mathrm{~d} x \geq \int_{\mathbb{R}^{d}}\left|\nabla_{x} u\right|^{2}\left(1+|x|^{2}\right)^{\alpha} \mathrm{d} x \\
& \geq \mathcal{C}_{\alpha, d} \int_{\mathbb{R}^{d}}\left|u^{\perp}\right|^{2}\left(1+|x|^{2}\right)^{\alpha-1} \mathrm{~d} x \geq \mathcal{C}_{\alpha, d} \int_{\mathbb{R}^{d}}\left|u^{\perp}\right|^{2} w \mathrm{~d} x,
\end{aligned}
$$

where $u=\bar{u}+u^{\perp}$ with $\bar{u}=0$ for $\alpha>\alpha^{*}, \bar{u}=$ constant, and

$$
\int_{\mathbb{R}^{d}} u^{\perp}\left(1+|x|^{2}\right)^{\alpha-1} \mathrm{~d} x=0 \quad \text { for } \quad \alpha<\alpha^{*} .
$$

This completes the proof for $\alpha>\alpha^{*}$. Otherwise, $\int_{\mathbb{R}^{d}} w \mathrm{~d} x<\infty$ holds, and the right hand side can be estimated as follows:

$$
\int_{\mathbb{R}^{d}}|u-\bar{u}|^{2} w \mathrm{~d} x \geq \inf _{\mu \in \mathbb{R}} \int_{\mathbb{R}^{d}}|u-\mu|^{2} w \mathrm{~d} x=\int_{\mathbb{R}^{d}} u^{2} w \mathrm{~d} x
$$

using the side condition $\int_{\mathbb{R}^{d}} u w \mathrm{~d} x=0$.

To get examples where macroscopic coercivity holds in the fast diffusion case, we apply Corollary 12 with $\alpha_{1}=-\beta /(1-m)$ and $\alpha_{2}=-\beta(2-m) /(1-m)$. Then

Assumption (H2.2): $\quad d \geq 3, \beta \geq 1$, and $m \neq(d-4) /(d-2)$

implies (H2). Note that the last condition is needed only for $\beta=1$. It will however be useful in the following. For proving the boundedness of $\mathrm{AT}(1-\Pi)$, a modified version of the framework of Section 2 can be used. Redefining

$$
W:=\left(1+|x|^{2}\right)^{(\beta-1) / 2},
$$

then $\nabla_{x} w_{1} / w_{0} \leq c W$ holds with the notation of Section 2 The result of Lemma 7 ,

$$
\left\|W \nabla_{x} u\right\|_{1}^{2} \geq \kappa^{\prime}\left\|W^{2} u\right\|_{0}^{2},
$$

is a direct consequence of the Hardy-Poincaré inequality with $\alpha=\beta-1+\beta /(m-1)$. Note that we have to require that $\alpha$ is different from $\alpha^{*}$. The proof of Lemma 8 uses assumption (2.5), which would together with (H2.2) require $\beta=1$ and therefore by (H0.2) $m>(d-4) /(d-2)$. This can be slightly improved by redoing the proof of Lemma 8 .

Lemma 13. Let (H2.2) hold. Then there exists a constant $\beta_{0}>1$, depending on $m$ and $d$, such that for $\beta<\beta_{0}$ the operator $\mathrm{AT}(1-\Pi)$ is bounded. 
Proof. As mentioned above, (4.3) follows from [2, Theorem 1]. Since, by (H2.2), $1 /(m-1) \neq \alpha^{*}$, also $\beta-1+\beta /(m-1) \neq \alpha^{*}$, if $\beta$ is close enough to 1 . According to [4], the explicit expression of the constant $\mathcal{C}_{\alpha, d}$ in (4.2) is a positive, continuous function of $\alpha$ for $\alpha<\alpha^{*}$ and $\alpha>\alpha^{*}$ and $\kappa^{\prime}=\kappa^{\prime}(\beta, m, d)>0$ in (4.3) can be chosen to be continuous with respect to $\beta$ at $\beta=1$.

As in the proof of Lemma 8 , we derive the inequality (2.10) and use

$$
w_{1}^{2}\left|\nabla_{x}\left(W^{2}\right)\right| \leq 2(\beta-1) w_{0} w_{1} W^{3}
$$

to estimate the last term by $2(\beta-1)\left\|u W^{2}\right\|_{0}\left\|W \nabla_{x} u\right\|_{1}$. With the help of (4.3), (2.10) implies

$$
\left\|W \nabla_{x} u\right\|_{1}^{2} \leq \frac{1}{\kappa^{\prime}}\left\|W \nabla_{x} u\right\|_{1}\left(\left\|u_{f}\right\|_{0}+2(\beta-1)\left\|W \nabla_{x} u\right\|_{1}\right) .
$$

By the continuity of $\kappa^{\prime}$, there exists $\beta_{0}(m, d)>1$ such that $2(\beta-1) / \kappa^{\prime}<1$ for $1 \leq \beta<\beta_{0}$, and for such a $\beta$ the result of Lemma 8 follows. This allows us to carry out the proof of Proposition [5, since assumption (2.6), which is used there, is satisfied.

As a consequence of this result, we formulate

Assumption (H4.3): $\quad \beta<\beta_{0}$ with $\beta_{0}(m, d)$ from Lemma 13 ,

Theorem 14. With the above notation, let $\mathrm{L}=\Pi-1$, and assume that $(\mathbf{H 0 . 2})$, (H2.2), and (H4.3) hold. Then solutions to (1.1) with initial data in $L^{2}(\mathrm{~d} \mu)$ decay exponentially to the global equilibrium given by

$$
F(x, v)=\left(\frac{1}{2}|v|^{2}+V(x)\right)^{-d / 2-1 /(1-m)-1}, \quad V(x)=\left(1+|x|^{2}\right)^{\beta} .
$$

This result is, to our knowledge, the first hypocoercivity result for a kinetic equation whose Gibbs state does not separate position and velocity variables.

\section{ACKNOWLEDGEMENTS}

This work was partially supported by the French-Austrian Amadeus project no. 13785UA, the ANR projects EVOL and CBDif-Fr, the Austrian Science Fund (project no. W8), and the European network DEASE. The second author thanks Cambridge University for their hospitality and acknowledges support from Award No. KUK-I1-007-43, funded by the King Abdullah University of Science and Technology (KAUST), as well as partial support from the ERC grant MATKIT.

\section{REFERENCES}

[1] Dominique Bakry, Franck Barthe, Patrick Cattiaux, and Arnaud Guillin, A simple proof of the Poincaré inequality for a large class of probability measures including the log-concave case, Electron. Commun. Probab. 13 (2008), 60-66, DOI 10.1214/ECP.v13-1352. MR2386063 (2009d:60039)

[2] Adrien Blanchet, Matteo Bonforte, Jean Dolbeault, Gabriele Grillo, and Juan-Luis Vázquez, Hardy-Poincaré inequalities and applications to nonlinear diffusions (English, with English and French summaries), C. R. Math. Acad. Sci. Paris 344 (2007), no. 7, 431-436, DOI 10.1016/j.crma.2007.01.011. MR2320246(2008b:35141)

[3] Adrien Blanchet, Matteo Bonforte, Jean Dolbeault, Gabriele Grillo, and Juan Luis Vázquez, Asymptotics of the fast diffusion equation via entropy estimates, Arch. Ration. Mech. Anal. 191 (2009), no. 2, 347-385, DOI 10.1007/s00205-008-0155-z. MR2481073 (2011d:35236) 
[4] M. Bonforte, J. Dolbeault, G. Grillo, and J. L. Vázquez, Sharp rates of decay of solutions to the nonlinear fast diffusion equation via functional inequalities, Proc. Natl. Acad. Sci. USA 107 (2010), no. 38, 16459-16464, DOI 10.1073/pnas.1003972107. MR2726546 (2011g:35187)

[5] Maria J. Cáceres, José A. Carrillo, and Thierry Goudon, Equilibration rate for the linear inhomogeneous relaxation-time Boltzmann equation for charged particles, Comm. Partial Differential Equations 28 (2003), no. 5-6, 969-989, DOI 10.1081/PDE-120021182. MR1986057 (2004g:82111)

[6] Carlo Cattaneo, Sulla conduzione del calore (Italian), Atti Sem. Mat. Fis. Univ. Modena 3 (1949), 83-101. MR0032898(11,362d)

[7] P. Degond, T. Goudon, and F. Poupaud, Diffusion limit for nonhomogeneous and nonmicro-reversible processes, Indiana Univ. Math. J. 49 (2000), no. 3, 1175-1198. MR.1803225 (2002a:35012)

[8] L. Desvillettes and C. Villani, On the trend to global equilibrium in spatially inhomogeneous entropy-dissipating systems: the linear Fokker-Planck equation, Comm. Pure Appl. Math. 54 (2001), no. 1, 1-42, DOI 10.1002/1097-0312(200101)54:1<1::AID-CPA1>3.0.CO;2Q. MR 1787105 (2001h:82079)

[9] L. Desvillettes and C. Villani, On the trend to global equilibrium for spatially inhomogeneous kinetic systems: the Boltzmann equation, Invent. Math. 159 (2005), no. 2, 245-316, DOI 10.1007/s00222-004-0389-9. MR2116276 (2005j:82070)

[10] Jean Dolbeault, Frédéric Hérau, Clément Mouhot, and Christian Schmeiser, Hypocoercivity in linear kinetic equations, In preparation, 2012.

[11] Jean Dolbeault, Peter Markowich, Dietmar Oelz, and Christian Schmeiser, Non linear diffusions as limit of kinetic equations with relaxation collision kernels, Arch. Ration. Mech. Anal. 186 (2007), no. 1, 133-158, DOI 10.1007/s00205-007-0049-5. MR2338354 (2009b:82085)

[12] Jean Dolbeault, Clément Mouhot, and Christian Schmeiser, Hypocoercivity for kinetic equations with linear relaxation terms (English, with English and French summaries), C. R. Math. Acad. Sci. Paris 347 (2009), no. 9-10, 511-516, DOI 10.1016/j.crma.2009.02.025. MR2576899 (2010j:35068)

[13] Klemens Fellner, Lukas Neumann, and Christian Schmeiser, Convergence to global equilibrium for spatially inhomogeneous kinetic models of non-micro-reversible processes, Monatsh. Math. 141 (2004), no. 4, 289-299, DOI 10.1007/s00605-002-0058-2. MR2053654 (2005f:82116)

[14] Robert T. Glassey, The Cauchy problem in kinetic theory, Society for Industrial and Applied Mathematics (SIAM), Philadelphia, PA, 1996. MR1379589 (97i:82070)

[15] Yan Guo, The Landau equation in a periodic box, Comm. Math. Phys. 231 (2002), no. 3, 391-434, DOI 10.1007/s00220-002-0729-9. MR.1946444 (2004c:82121)

[16] Yan Guo, The Vlasov-Poisson-Boltzmann system near Maxwellians, Comm. Pure Appl. Math. 55 (2002), no. 9, 1104-1135, DOI 10.1002/cpa.10040. MR.1908664(2003b:82050)

[17] Yan Guo, Classical solutions to the Boltzmann equation for molecules with an angular cutoff, Arch. Ration. Mech. Anal. 169 (2003), no. 4, 305-353, DOI 10.1007/s00205-003-0262-9. MR2013332(2004i:82054)

[18] Yan Guo, The Vlasov-Maxwell-Boltzmann system near Maxwellians, Invent. Math. 153 (2003), no. 3, 593-630, DOI 10.1007/s00222-003-0301-z. MR2000470 (2004m:82123)

[19] Yan Guo, The Boltzmann equation in the whole space, Indiana Univ. Math. J. 53 (2004), no. 4, 1081-1094, DOI 10.1512/iumj.2004.53.2574. MR.2095473 (2005g:35028)

[20] Frédéric Hérau and Karel Pravda-Starov, Anisotropic hypoelliptic estimates for Landau-type operators (English, with English and French summaries), J. Math. Pures Appl. (9) 95 (2011), no. 5, 513-552, DOI 10.1016/j.matpur.2010.11.003. MR2786222 (2012c:35074)

[21] Frédéric Hérau, Hypocoercivity and exponential time decay for the linear inhomogeneous relaxation Boltzmann equation, Asymptot. Anal. 46 (2006), no. 3-4, 349-359. MR2215889 (2007b:35044)

[22] Frédéric Hérau and Francis Nier, Isotropic hypoellipticity and trend to equilibrium for the Fokker-Planck equation with a high-degree potential, Arch. Ration. Mech. Anal. 171 (2004), no. 2, 151-218, DOI 10.1007/s00205-003-0276-3. MR2034753(2005f:82085)

[23] Michael Hitrik and Karel Pravda-Starov, Semiclassical hypoelliptic estimates for nonselfadjoint operators with double characteristics, Comm. Partial Differential Equations 35 (2010), no. 6, 988-1028, DOI 10.1080/03605301003717092. MR2753626 (2011m:35050) 
[24] Shuichi Kawashima, The Boltzmann equation and thirteen moments, Japan J. Appl. Math. 7 (1990), no. 2, 301-320, DOI 10.1007/BF03167846. MR.1057534 (91i:82020)

[25] Ming-Yi Lee, Tai-Ping Liu, and Shih-Hsien Yu, Large-time behavior of solutions for the Boltzmann equation with hard potentials, Comm. Math. Phys. 269 (2007), no. 1, 17-37, DOI 10.1007/s00220-006-0108-z. MR2274461 (2007k:82115)

[26] Tai-Ping Liu and Shih-Hsien Yu, Boltzmann equation: micro-macro decompositions and positivity of shock profiles, Comm. Math. Phys. 246 (2004), no. 1, 133-179, DOI 10.1007/s00220003-1030-2. MR2044894(2005f:82101)

[27] Tai-Ping Liu and Shih-Hsien Yu, Initial-boundary value problem for one-dimensional wave solutions of the Boltzmann equation, Comm. Pure Appl. Math. 60 (2007), no. 3, 295-356, DOI 10.1002/cpa.20172. MR2284213 (2009b:35025)

[28] Clément Mouhot and Lukas Neumann, Quantitative perturbative study of convergence to equilibrium for collisional kinetic models in the torus, Nonlinearity 19 (2006), no. 4, 969998, DOI 10.1088/0951-7715/19/4/011. MR2214953(2007c:82032)

[29] A. Pazy, Semigroups of linear operators and applications to partial differential equations, Applied Mathematical Sciences, vol. 44, Springer-Verlag, New York, $1983 . \quad$ MR710486 (85g:47061)

[30] Arne Persson, Bounds for the discrete part of the spectrum of a semi-bounded Schrödinger operator, Math. Scand. 8 (1960), 143-153. MR0133586 (24 \#A3412)

[31] Robert M. Strain and Yan Guo, Stability of the relativistic Maxwellian in a collisional plasma, Comm. Math. Phys. 251 (2004), no. 2, 263-320, DOI 10.1007/s00220-004-1151-2. MR2100057(2005m:82155)

[32] Robert M. Strain and Yan Guo, Almost exponential decay near Maxwellian, Comm. Partial Differential Equations 31 (2006), no. 1-3, 417-429, DOI 10.1080/03605300500361545. MR2209761 (2006m:82042)

[33] Robert M. Strain and Yan Guo, Exponential decay for soft potentials near Maxwellian, Arch. Ration. Mech. Anal. 187 (2008), no. 2, 287-339, DOI 10.1007/s00205-007-0067-3. MR.2366140 (2008m:82008)

[34] Seiji Ukai, On the existence of global solutions of mixed problem for non-linear Boltzmann equation, Proc. Japan Acad. 50 (1974), 179-184. MR0363332 (50 \#15770)

[35] Cédric Villani, Hypocoercivity, Mem. Amer. Math. Soc. 202 (2009), no. 950, iv+141, DOI 10.1090/S0065-9266-09-00567-5. MR2562709 (2011e:35381)

[36] Shih-Hsien Yu, The development of the Green's function for the Boltzmann equation, J. Stat. Phys. 124 (2006), no. 2-4, 301-320, DOI 10.1007/s10955-006-9064-4. MR2264611 (2007i:82072)

Ceremade (UmR CNRS no. 7534), Université Paris-Dauphine, Place de lattre de Tassigny, 75775 Paris Cedex 16, France

E-mail address: dolbeaul@ceremade.dauphine.fr

Centre for Mathematical Sciences, University of Cambridge, Wilberforce Road, CAmbridge CB3 0WA, United Kingdom

E-mail address: C.Mouhot@dpmms.cam.ac.uk

Fakultät für Mathematik, Universität Wien, Nordbergstrasse 15, 1090 Wien, AusTRIA

E-mail address: Christian.Schmeiser@univie.ac.at 